\title{
An accelerated Newton method of high-order convergence for solving a class of weakly nonlinear complementarity problems
}

\author{
Ya-Jun Xie ${ }^{\mathrm{a}, \mathrm{b}}$, Na Huang ${ }^{\mathrm{a}, \mathrm{c}}$, Chang-Feng Ma ${ }^{\mathrm{a}, *}$ \\ ${ }^{a}$ College of Mathematics and Informatics, Fujian Key Laboratory of Mathematical Analysis and Applications, Fujian Normal University, \\ Fuzhou 350117, P. R. China. \\ ${ }^{b}$ Department of Mathematics and Physics, Fujian Jiangxia University, Fuzhou 350108, P. R. China. \\ ${ }^{c}$ Academy of Mathematics and Systems Science, Chinese Academy of Sciences, Beijing 100190, P. R. China.
}

Communicated by Y. Z. Chen

\begin{abstract}
In this paper, by extending the classical Newton method, we investigate an accelerated Newton iteration method (ANIM) with high-order convergence for solving a class of weakly nonlinear complementarity problems which arise from the discretization of free boundary problems. Theoretically, the performance of high-order convergence is analyzed in details. Some numerical experiments demonstrate the efficiency of the presented method. (C)2017 All rights reserved.
\end{abstract}

Keywords: Weakly nonlinear complementarity problems, high-order convergence, the modulus-based nonlinear function, convergence analysis, numerical experience.

2010 MSC: $65 \mathrm{H} 10,90 \mathrm{C} 33$.

\section{Introduction}

In this paper we discuss the following weakly nonlinear complementarity problems, to find a vector $u \in \mathbb{R}^{n}$ such that

$$
\left\{\begin{array}{l}
u \geqslant 0 \\
w:=A u+\Phi(u) \geqslant 0 \\
w^{\top} u=0
\end{array}\right.
$$

where $A \in \mathbb{R}^{n \times n}$ is given large, sparse and real matrix, $\Phi(u): \mathbb{R}^{n} \rightarrow \mathbb{R}^{n}$ is a Lipschitz continuous nonlinear function.

The weakly nonlinear complementarity problems have been investigated extensively owing to various scientific and engineering applications, such as operations research, economic equilibrium, and engineering design. When $\Phi(u)=q$ in formula (1.1), the weakly nonlinear complementarity problems reduce to

\footnotetext{
*Corresponding author

Email address: macf@f jnu.edu.cn (Chang-Feng Ma)
}

doi:10.22436/jnsa.010.09.24 
the following linear complementarity problems:

$$
\left\{\begin{array}{l}
u \geqslant 0 \\
w:=A u+q \geqslant 0, \\
w^{\top} u=0
\end{array}\right.
$$

where $A \in \mathbb{R}^{n \times n}$ and $q \in \mathbb{R}^{n}$ are given real matrix and real vector, respectively. The problems are often abbreviated as $\operatorname{LCP}(q, A)$. As the special case, it frequently arises in Nash equilibrium point of a bimatrix game, the contract problem, and the free boundary problem for journal bearings, see [11, 21, 23]. For examples, in [21], Lemke investigated firstly a solution for linear complementarity problem. Moreover, Scarf obtained the approximation of fixed points of a continuous mapping [26]. The relationships between the linear complementarity problem and the fixed points problem are well described by Eaves et al. $[14,15]$.

A variety of efficient methods were proposed to solve (1.2), especially, when the system matrix $A$ is large and sparse. Such as, the projected successive over-relaxation iteration [12] and the general fixedpoint iterations [28]. In recent years, there has been an increasing interest in solving (1.2) by applying the so-called non-interior continuation smoothing methods. About the matrix splitting iterations approaches, Bai et al. have established many fruitful research results [3, 5, 7, 9]. Especially, in [3], Bai presented the Modulus-based matrix splitting iteration scheme which is a powerful method for solving (1.2). On matrix multisplitting iteration aspects, also, there are many useful papers were introduced to solve (1.2), see [1, 2, $4,6,8]$ for more details. Many kinds of accelerated modulus-based matrix splitting iteration versions are also developed, see [30, 31]. Moreover, the Modulus-based synchronous multisplitting iteration methods for (1.2) are considered in [9]. Inspired by [9], Cvetković and Kostić did not depend on the assumption of parameter constraint, however, ameliorated the field of convergence [13]. Recently, Foutayeni et al. proposed an efficient hybrid method for solving (1.2) by using the vector divisions and the secant method in [16].

For the weakly nonlinear complementarity problems, Sun and Zeng in [27] presented a monotone semismooth Newton type method for these problems. Under some appropriate conditions, the method reduces to semismooth Newton method. As is known that semismooth (or smooth) Newton method is pretty effective for some nonsmooth (or smooth) equations, which arises from the complementarity problem, the nonlinear programming problem, the maximal monotone operator problem, the discretization problem of partial differential equations, and the variational inequality problem, etc, see [10, 20, 25] for a more detailed discuss. These approaches are attractive due to the rapid convergence for any sufficiently right initial guess. Some merit functions, such as squared norm merit function, are often exploited in order to ensure that the global convergence of semismooth Newton method, see [24] and references therein. Recently, in [18], Huang and Ma investigated a modulus-based matrix splitting algorithms for a class of weakly nonlinear complementarity problems, which is shown as a quite valid technique for the problems (1.1).

Motivated by these works, via introducing a smooth equation and some reasonable equivalent reformulations, we present an accelerated Newton iteration method with high-order convergence rate for solving a class of large-scale weakly nonlinear complementarity problem, which make full use of the superiority of second-order convergence rate of the classical Newton method.

To characterize the contexts, the following notations are introduced throughout the paper: let $\mathbb{N}_{k}=$ $\{1,2, \cdots, k\}$ stand for the set of first $k$ positive integers. For $x \in \mathbb{R}^{n},\|x\|$ denotes the Euclidean norm. Given two real $n \times m$ matrices $A=\left(a_{i j}\right)$ and $B=\left(b_{i j}\right)$, we write $A \geqslant B$ (or $A>B$ ) if $a_{i j} \geqslant b_{i j}$ (or $a_{i j}>b_{i j}$ ) holds for all $i \in \mathbb{N}_{n}$ and $j \in \mathbb{N}_{m}$. We use $|A|$ and $\rho(A)$ to refer to the absolute value and spectral radius of the matrix $A \in \mathbb{R}^{n \times m}$, respectively. For the differentiable function $F(x), F^{\prime}(x)$ is referred to the Jacobi matrix of the function $F(x)$. For a nonsingular matrix $A, A^{-1}$ stands for the inverse matrix. In addition, the matrix $\operatorname{Diag}\left\{a_{1}, a_{2}, \cdots, a_{n}\right\}$ denotes the diagonal matrix, where $a_{i}\left(i \in \mathbb{N}_{n}\right)$ is the element of the principal diagonal. 
The remainder of this paper is organized as follows. In Section 2, an accelerated Newton iteration method (ANIM) with high-order convergence rate is proposed for solving a class of weakly nonlinear complementarity problems (1.1). A detailed discussion on the performance and rate of convergence of the ANIM is given in Section 3. In Section 4, some numerical tests are provided to demonstrate the superiority of the presented method to some current efficient approaches. Finally, some concluding remarks are given in Section 5.

\section{The accelerated Newton method}

In this section, we first give some valuable conclusions which are important to the equivalent reformulation for the weakly nonlinear complementarity problem. Moreover, these results contribute significantly to the analysis of the convergence rate of the accelerated Newton iteration method.

Lemma 2.1. The formula (1.1) is equivalent to the system of nonlinear equation

$$
(I+A) x+(I-A)|x|-\Phi(|x|-x)=0,
$$

where $\mathrm{A}$ and $\Phi(\cdot)$ are given matrix and Lipschitz continuous nonlinear function in (1.1), respectively, I is the identity matrix with appropriate dimension, and $x \in \mathbb{R}^{n}$ is the vector to be determined.

Proof. First, let $x$ be the solution of Eq. (2.1). Then we get

$$
|x|+x=A(|x|-x)+\Phi(|x|-x) .
$$

Set

$$
w:=|x|+x, u:=|x|-x .
$$

It follows from (2.2) and (2.3) that

$$
w=A u+\Phi(u), \quad w \geqslant 0, u \geqslant 0, w^{\top} u=0,
$$

it implies that $u$ is the solution of (1.1).

In addition, we assume that $u$ is the solution of (1.1). It is evidently that

$$
w \geqslant 0, \quad u \geqslant 0, \quad w^{\top} u=0,
$$

where $w=A u+\Phi(u)$.

Analogously, we can set

$$
w:=|x|+x, u:=|x|-x .
$$

It yields that

$$
x=\frac{1}{2}(w-u)
$$

satisfies the Eq. (2.1).

Next, we introduce the smooth function $F: \mathbb{R}^{n+1} \rightarrow \mathbb{R}^{n}$ by

$$
F(z):=(I-A) \sqrt{x^{2}+\varepsilon^{2} \mathbf{e}}+(I+A) x-\Phi\left(\sqrt{x^{2}+\varepsilon^{2} \mathbf{e}}-x\right),
$$

where $z=\left(\varepsilon, x^{\top}\right)^{\top} \in \mathbb{R}^{n+1}, \varepsilon$ is a positive variable, $\mathbf{e}=(1,1, \cdots, 1)^{\top} \in \mathbb{R}^{n}$,

$$
\sqrt{x^{2}+\varepsilon^{2} \mathbf{e}}:=\left[\sqrt{x_{1}^{2}+\varepsilon^{2}}, \sqrt{x_{2}^{2}+\varepsilon^{2}}, \cdots, \sqrt{x_{n}^{2}+\varepsilon^{2}}\right]^{\top} \in \mathbb{R}^{n} .
$$

Furthermore, we define the nonlinear and differentiable function

$$
\Psi(z):=\left(\begin{array}{c}
\varepsilon \\
F(z)
\end{array}\right) \in \mathbb{R}^{n+1} .
$$


Lemma 2.2. The formula (1.1) is equivalent to the nonlinear system

$$
\Psi(z)=0,
$$

where $\Psi(z)$ is defined by $(2.6), z=\left(\varepsilon, x^{\top}\right)^{\top} \in \mathbb{R}^{n+1}$.

Proof. This result can be obtained easily from the formula (2.4) and Lemma 2.1. So we omit it.

Lemma 2.3. The Jocabian matrix of formula (2.6) is

$$
\Psi^{\prime}(z)=\left(\begin{array}{cc}
1 & 0 \\
\left(I-A-\Psi_{\tilde{z}}^{\prime}(\widetilde{z})\right) g_{\varepsilon} & \left(I-A-\Psi_{\tilde{z}}^{\prime}(\widetilde{z})\right) D_{x}+\Psi_{\widetilde{z}}^{\prime}(\widetilde{z})+A+I
\end{array}\right) \in \mathbb{R}^{(n+1) \times(n+1),}
$$

where $g_{\varepsilon}=\left(g_{1}, g_{2}, \cdots, g_{n}\right)^{\top} \in \mathbb{R}^{n}, g_{i}=\frac{\varepsilon}{\sqrt{x_{i}^{2}+\varepsilon^{2}}}, D_{x}=\operatorname{Diag}\left(d_{1}, d_{2}, \cdots, d_{n}\right) \in \mathbb{R}^{n \times n}, d_{i}=\frac{x_{i}}{\sqrt{x_{i}^{2}+\varepsilon^{2}}}$ $\left(i \in \mathbb{N}_{n}\right.$ ), and $\widetilde{z}:=\sqrt{\chi^{2}+\varepsilon^{2} \mathbf{e}}-x, \mathbf{0} \in \mathbb{R}^{n}$ denotes the zero vector.

Proof. By implementing derivative for variable $z$ on both sides of the Eq. (2.6), we have

$$
\Psi^{\prime}(z)=\left(\begin{array}{cc}
1 & 0 \\
F_{\varepsilon}^{\prime} & F_{x}^{\prime}
\end{array}\right)
$$

It follows from formulas (2.4) and (2.5) that

$$
\begin{aligned}
\mathrm{F}_{\varepsilon}^{\prime}= & (\mathrm{I}-\mathrm{A})\left(\frac{\varepsilon}{\sqrt{x_{1}^{2}+\varepsilon^{2}}}, \frac{\varepsilon}{\sqrt{x_{2}^{2}+\varepsilon^{2}}}, \cdots, \frac{\varepsilon}{\sqrt{x_{n}^{2}+\varepsilon^{2}}}\right)^{\top} \\
& -\Psi_{\widetilde{z}}^{\prime}(\widetilde{z})\left(\frac{\varepsilon}{\sqrt{x_{1}^{2}+\varepsilon^{2}}}, \frac{\varepsilon}{\sqrt{x_{2}^{2}+\varepsilon^{2}}}, \cdots, \frac{\varepsilon}{\sqrt{x_{\mathfrak{n}}^{2}+\varepsilon^{2}}}\right)^{\top}=\left(I-A-\Psi_{\widetilde{z}}^{\prime}(\widetilde{z})\right) g_{\varepsilon} .
\end{aligned}
$$

As $\widetilde{z}_{x}^{\prime}=D_{x}-\mathrm{I}$, we get

$$
F_{x}^{\prime}=(I-A) D_{x}+A+I-\Psi_{\widetilde{z}}^{\prime}(\widetilde{z}) \cdot \widetilde{z}_{x}^{\prime}=\left(I-A-\Psi_{\widetilde{z}}^{\prime}(\widetilde{z})\right) D_{x}+\Psi_{\widetilde{z}}^{\prime}(\widetilde{z})+I+A,
$$

which completes the proof.

Next, we will give the accelerated Newton iteration method for the nonlinear smooth system (2.7). The detailed description is shown as follows.

Algorithm 2.4 (The Accelerated Newton iteration method (ANIM) for (2.7)).

Step 1. Give the initial guess $z^{0}=\left(\varepsilon_{0},\left(x^{0}\right)^{\top}\right)^{\top}$, the matrix $A$, vector $q$ and any small positive numbers $\sigma_{1}, \sigma_{2} \in(0,1)$, and preset a positive integer $m \geqslant 2$. Set $k:=0$.

Step 2. Calculate $\Psi\left(z^{k}\right)$, the Jacobian matrix $\Psi^{\prime}\left(z^{k}\right)$, and the inverse matrix $A_{k}:=\left(\Psi^{\prime}\left(z^{k}\right)\right)^{-1}$.

Step 3. Let $z^{k, 1}=z^{k}, j:=1$.

Step 4. Compute $\Psi\left(z^{k, j}\right)$, update the vector sequence

$$
z^{k, j+1}=z^{k, j}-A_{k} b
$$

then evaluate $\Psi\left(z^{k, j+1}\right)$, where $b=\Psi\left(z^{k, j}\right)$.

Step 5. Let $j:=j+1, z^{k, j}:=z^{k, j+1}, \Psi\left(z^{k, j}\right):=\Psi\left(z^{k, j+1}\right), y:=A_{k} b$. When $j=m$, return to Step 6, otherwise go to Step 4.

Step 6. When $\|y\|<\sigma_{1}$ or $\left\|\Psi\left(z^{k, m}\right)\right\|<\sigma_{2}$, let $z^{*}=z^{k, m}$, otherwise put $k:=k+1$, return to Step 3 . 
Actually, the Accelerated Newton iteration method (ANIM) can also be written with the iteration scheme as follows:

$$
\left\{\begin{array}{l}
z^{k, 0}=z^{k} \\
z^{k, j}=z^{k, j-1}-\left(\Psi^{\prime}\left(z^{k}\right)\right)^{-1} \Psi\left(z^{k, j-1}\right), j=1,2 \cdots, m, \\
z^{k+1}=z^{k, m}, \quad k=0,1,2, \cdots .
\end{array}\right.
$$

Remark 2.5. By Lemma 2.2, we conclude that the iteration solution $z^{*}$ generated by Algorithm 2.4 is also the solution $u^{*}$ of formula (1.1).

Remark 2.6. The update of the parameter $\varepsilon_{k}$ can be selected with $\varepsilon_{k}=\varepsilon_{k-1}^{m}$. Because the positive integer $\mathrm{m}$ is chosen at least greater than or equal to 2 in Algorithm 2.4, the positive sequence $\left\{\varepsilon_{\mathrm{k}}\right\}_{0}^{\infty}$ monotonically decreases and tends to zero.

Remark 2.7. If we set $m=1$, then the ANIM will retrograde to the classical Newton iterative method.

\section{The analysis of convergence}

In this section, we will give the analysis of convergence of the accelerated Newton iteration method (ANIM). To this end, we first give a major definition and some significant lemmas.

Definition 3.1 ([19]). Let $F: D \subset \mathbb{R}^{n} \rightarrow \mathbb{R}^{n}, x^{*} \in D$ be the solution of system $F(x)=0$. There is a region $S \subset D$ for the point $x^{*}$. For any initial approximation $x^{0} \in S$, if the iteration sequence $\left\{x^{k}, k=0,1, \cdots\right\}$ is always well-defined and converges to the solution $x^{*}$, we call it an attractive point of the iteration sequence.

In view of the classical Newton iteration at least has two-order convergence rate, we have the following results. For more details see [19] and references therein.

Lemma 3.2. Assume $\mathrm{F}: \mathrm{D} \subset \mathbb{R}^{\mathrm{n}} \rightarrow \mathbb{R}^{\mathrm{n}}$ is Fréchet differentiable on the open interval of $\mathrm{S}_{0} \in \mathrm{D}$ and $\mathrm{F}^{\prime}\left(\mathrm{x}^{*}\right)$ is nonsingular, where $x^{*}$ is the solution of system $\mathrm{F}(\mathrm{x})=0$. Then, the mapping $\mathrm{G}(\mathrm{x})=\mathrm{x}-\mathrm{F}^{\prime}(\mathrm{x})^{-1} \mathrm{~F}(\mathrm{x})$ is well-defined on $\mathrm{S}$ for the closed sphere $\mathrm{S}=\overline{\mathrm{S}}\left(\mathrm{x}^{*}, \delta\right) \subset \mathrm{S}_{0}$. Moreover, if the inequality

$$
\left\|F^{\prime}(x)-F^{\prime}\left(x^{*}\right)\right\| \leqslant \beta\left\|x-x^{*}\right\|
$$

holds, where $\beta$ is a constant and $x \in S$, then the classical Newton iteration method has at least the two-order convergence rate.

Lemma 3.3. Assume $\mathrm{F}: \mathrm{D} \subset \mathbb{R}^{\mathrm{n}} \rightarrow \mathbb{R}^{\mathrm{n}}$ is Fréchet differentiable on the fixed point $\mathrm{x}^{*} \in \operatorname{int}(\mathrm{D})$ and the spectral radius

$$
\rho\left(F^{\prime}\left(x^{*}\right)\right)=\sigma<1 .
$$

Then $x^{*}$ is the attractive point of the iterative sequence $x^{k+1}=F\left(x^{k}\right)(k=0,1, \cdots)$ for the open sphere $S=$ $\mathrm{S}\left(\mathrm{x}^{*}, \delta\right) \subset \mathrm{D}$ and any initial guess $\mathrm{x}^{0} \in \mathrm{S}$.

Lemma 3.4. Assume $\mathrm{F}: \mathrm{D} \subset \mathbb{R}^{\mathrm{n}} \rightarrow \mathbb{R}^{\mathrm{m}}$ is continuous and differentiable on the convex set $\mathrm{D}_{0} \subset \mathrm{D}$ and it satisfies

$$
\left\|F^{\prime}(u)-F^{\prime}(v)\right\| \leqslant \beta\|u-v\|^{p}, u, v \in D_{0} .
$$

Then there holds

$$
\left\|F(y)-F(x)-F^{\prime}(x)(y-x)\right\| \leqslant \frac{\beta}{1+p}\|y-x\|^{1+p}, x, y \in D_{0},
$$

where $\beta \geqslant 0, p \geqslant 0$ are constants. 
Next, we will give the convergence of the accelerated Newton iteration method. The detailed result and the proof process are shown as follows.

Theorem 3.5. Let $\Psi: D \subset \mathbb{R}^{n+1} \rightarrow \mathbb{R}^{n+1}$ be Fréchet differentiable on the circle region of $\mathrm{S}\left(z^{*}, \delta\right) \in \mathrm{D}$ and $\Psi^{\prime}\left(z^{*}\right)$ be nonsingular. There is $\beta>0$ for each $z \in S$ such that

$$
\left\|\Psi^{\prime}(z)-\Psi^{\prime}\left(z^{*}\right)\right\| \leqslant \beta\left\|z-z^{*}\right\|
$$

holds. Then $z^{*}$ is the attractive point of the iterative sequence $\left\{z^{k}\right\}_{0}^{\infty}$ generated by Algorithm 2.4 and

$$
\left\|z^{\mathrm{k}+1}-z^{*}\right\| \leqslant \mathrm{L}_{2}\left\|z^{\mathrm{k}}-z^{*}\right\|^{\mathrm{m}+1},
$$

where $z^{*}$ is the solution of $\Psi(z)=0$, and $\mathrm{L}_{2}$ is a constant independent of iteration number $\mathrm{k}$.

Proof. Firstly, we consider the iterative formula (2.8) with the case $m=2$. That is to say,

$$
z^{k+1}=z^{k}-\left(\Psi^{\prime}\left(z^{k}\right)\right)^{-1}\left[\Psi\left(z^{k}\right)-\Psi\left(z^{k}-\left(\Psi^{\prime}\left(z^{k}\right)\right)^{-1} \Psi\left(z^{k}\right)\right)\right], \quad k=0,1, \cdots
$$

According to Lemma 3.2, $\mathrm{P}(z):=z-\left(\Psi^{\prime}(z)\right)^{-1} \Psi(z)$ is well-defined on $\mathrm{S}_{1}:=\overline{\mathrm{S}}\left(z^{*}, \delta_{1}\right) \subset \mathrm{S}$ and

$$
\left\|\mathrm{P}(z)-z^{*}\right\| \leqslant \xi\left\|z-z^{*}\right\|^{2}, \quad z \in \mathrm{S}_{1}
$$

where $\xi$ is a positive constant. Therefore, the mapping $M(z)=P(z)-\left(\Psi^{\prime}(z)\right)^{-1} \Psi(P(z))$ is well-defined on the closed sphere $S_{2}=\bar{S}\left(z^{*}, \delta_{2}\right) \subset S_{1}$, where $\delta_{2} \leqslant \frac{\delta_{1}}{\xi}$. Notice that $\Psi\left(z^{*}\right)=0$, so $P\left(z^{*}\right)=z^{*}$. By simple calculation, we have

$$
\mathrm{P}^{\prime}(z)=\mathrm{I}+\left(\Psi^{\prime}(z)\right)^{-2} \Psi^{\prime \prime}(z) \Psi(z)-\left(\Psi^{\prime}(z)\right)^{-1} \Psi^{\prime}(z)=\left(\Psi^{\prime}(z)\right)^{-2} \Psi^{\prime \prime}(z) \Psi(z),
$$

hence $\mathrm{P}^{\prime}\left(z^{*}\right)=0$. Moreover,

$$
M^{\prime}\left(z^{*}\right)=\mathrm{P}^{\prime}\left(z^{*}\right)+\left(\Psi^{\prime}\left(z^{*}\right)\right)^{-2} \Psi^{\prime \prime}\left(z^{*}\right) \Psi\left(\mathrm{P}\left(z^{*}\right)\right)-\left(\Psi^{\prime}\left(z^{*}\right)\right)^{-1} \Psi^{\prime}\left(\mathrm{P}\left(z^{*}\right)\right) \mathrm{P}^{\prime}\left(z^{*}\right)=0,
$$

where the last equality holds due to $\Psi\left(\mathrm{P}\left(z^{*}\right)\right)=\Psi\left(z^{*}\right)=0$. This implies $\rho\left(M^{\prime}\left(z^{*}\right)\right)=0<1$. Then, by Lemma 3.3, it yields that $z^{*}$ is the attractive point of (3.2).

In addition, notice that the nonsingularity of $\Psi^{\prime}\left(z^{*}\right)$, so $\left\|\left(\Psi^{\prime}(z)\right)^{-1}\right\| \leqslant \zeta$ for $z \in S_{2}$. Then it follows from Lemma 3.4 and the hypothesis that

$$
\begin{aligned}
\left\|\mathrm{M}(z)-z^{*}\right\| & \leqslant\left\|\left(\Psi^{\prime}(z)\right)^{-1}\right\|\left\|\Psi^{\prime}(z)\left[\mathrm{P}(z)-z^{*}\right]-\Psi(\mathrm{P}(z))\right\| \\
& \leqslant \zeta\left[\left\|\Psi(\mathrm{P}(z))-\Psi\left(z^{*}\right)-\Psi^{\prime}\left(z^{*}\right)\left(\mathrm{P}(z)-z^{*}\right)\right\|+\left\|\left(\Psi^{\prime}\left(z^{*}\right)-\Psi^{\prime}(z)\right)\left(\mathrm{P}(z)-z^{*}\right)\right\|\right] \\
& \leqslant \zeta\left[\frac{1}{2} \beta\left\|\mathrm{P}(z)-z^{*}\right\|^{2}+\beta\left\|z-z^{*}\right\|\left\|\mathrm{P}(z)-z^{*}\right\|\right] \\
& \leqslant \beta \zeta\left[\frac{1}{2} \xi^{2}\left\|z-z^{*}\right\|+\xi\right]\left\|z-z^{*}\right\|^{3} \\
& \leqslant \beta \zeta \xi\left[\frac{1}{2} \xi \delta_{2}+1\right]\left\|z-z^{*}\right\|^{3} .
\end{aligned}
$$

Set $\mathrm{L}_{0}:=\beta \zeta \xi\left[\frac{1}{2} \xi \delta_{2}+1\right]$, obviously, it is a constant independent of the iteration number $k$.

If we select $z^{k+1}=M\left(z^{k}\right)$ for the left side of the inequality (3.3), then by some simple manipulations, it gives

$$
\left\|z^{\mathrm{k}+1}-z^{*}\right\| \leqslant \mathrm{L}_{0}\left\|z^{\mathrm{k}}-z^{*}\right\|^{3}
$$

which implies (3.1) holds for $m=2$. 
Here, we state that the iterative scheme (2.8) at least has $m+1$ order convergence rate. The conclusion will be confirmed by mathematical induction.

Observing that (3.3), it has the result

$$
\left\|z^{k, 2}-z^{*}\right\| \leqslant \mathrm{L}_{0}\left\|z^{\mathrm{k}}-z^{*}\right\|^{3}=\mathrm{O}\left(\left\|z^{\mathrm{k}}-z^{*}\right\|^{3}\right) .
$$

Moreover, we suppose that

$$
\left\|z^{k, m-1}-z^{*}\right\| \leqslant \mathrm{L}_{1}\left\|z^{k}-z^{*}\right\|^{m}=\mathrm{O}\left(\left\|z^{k}-z^{*}\right\|^{m}\right)
$$

holds. Next, we will show the statement

$$
\left\|z^{k, m}-z^{*}\right\| \leqslant L_{2}\left\|z^{k}-z^{*}\right\|^{m+1}=\mathrm{O}\left(\left\|z^{k}-z^{*}\right\|^{m+1}\right),
$$

where $\mathrm{L}_{1}, \mathrm{~L}_{2}$ are the constants independent of iteration number $\mathrm{k}$.

Combining Lemma 3.4 and (3.4), and also noting that (2.8), we have

$$
\begin{aligned}
&\left\|z^{\mathrm{k}, \mathrm{m}}-z^{*}\right\| \leqslant\left\|\left(\Psi^{\prime}\left(z^{\mathrm{k}}\right)\right)^{-1}\right\|\left[\left\|\Psi\left(z^{\mathrm{k}, \mathrm{m}-1}\right)-\Psi\left(z^{*}\right)-\Psi^{\prime}\left(z^{*}\right)\left(z^{\mathrm{k}, \mathrm{m}-1}-z^{*}\right)\right\|\right. \\
&\left.+\left\|\left(\Psi^{\prime}\left(z^{*}\right)-\Psi^{\prime}\left(z^{\mathrm{k}}\right)\right)\left(z^{\mathrm{k}, \mathrm{m}-1}-z^{*}\right)\right\|\right] \\
& \leqslant \zeta\left[\frac{1}{2} \beta\left\|z^{\mathrm{k}, \mathrm{m}-1}-z^{*}\right\|^{2}+\beta\left\|z^{\mathrm{k}}-z^{*}\right\|\left\|z^{\mathrm{k}, \mathrm{m}-1}-z^{*}\right\|\right] \\
& \leqslant \zeta \beta\left[\frac{1}{2} \mathrm{~L}_{1}^{2}\left\|z^{\mathrm{k}}-z^{*}\right\|^{\mathrm{m}-1}+\mathrm{L}_{1}\right]\left\|z^{\mathrm{k}}-z^{*}\right\|^{\mathrm{m}+1} \\
& \leqslant \zeta \beta\left[\frac{1}{2} \mathrm{~L}_{1}^{2} \delta_{2}^{\mathrm{m}-1}+\mathrm{L}_{1}\right]\left\|z^{\mathrm{k}}-z^{*}\right\|^{\mathrm{m}+1} \\
&=\mathrm{L}_{2}\left\|z^{\mathrm{k}}-z^{*}\right\|^{\mathrm{m}+1}=\mathrm{O}\left(\left\|z^{\mathrm{k}}-z^{*}\right\|^{\mathrm{m}+1}\right),
\end{aligned}
$$

where $\mathrm{L}_{2}:=\zeta \beta\left[\frac{1}{2} \mathrm{~L}_{1}^{2} \delta_{2}^{\mathrm{m}-1}+\mathrm{L}_{1}\right]$. This concludes the proof of the theorem.

\section{Numerical experiments}

In this section, some numerical examples are discussed to illustrate the effectiveness and advantages of the proposed ANIM for solving the weakly nonlinear complementarity problem. We compare the performance of the ANIM against the Fischer-based semismooth Newton method which we denote FBSN (see $[17,22])$, the cosh-based smoothing Newton method which we name CBSN (see [29]), the modulus-based matrix splitting algorithms which we call MMSA (see [18]) by the iteration step (denoted as 'IT'), elapsed CPU time in seconds (denoted as 'CPU'), and residual error (denoted as 'RES'). In actual computations, all runs are terminated when the current iteration satisfies

$$
\text { RES }:=\left\|\Psi\left(z^{k}\right)\right\|<10^{-10}
$$

or if the number of iteration exceeds the prescribed iteration steps $k_{\max }$. The numerical experiments have been carried out by MATLAB R2011b 7.1.3 on a PC equipped with an Intel(R) Core(TM) i7-2670QM, CPU running at 2.20GHZ with $8 \mathrm{~GB}$ of RAM in Windows 7 operating system.

After the previous analysis, we know that the ANIM can be considered as the acceleration version on the basis of the smoothing Newton method. Hence, high order convergence is the advantage of the ANIM. In fact, if we set $m=1$, the ANIM will reduce to the classical Newton method. We know, however, the larger $\mathrm{m}$ may lead to consume more CPU time on account of increasing the number of inner iterations. We usually only select $m=2$ in concrete examples, which also can ensure the rapid convergence rate in Algorithm 2.4. To further confirm this judgment, we will put into effect by the following examples.

In these examples, we choose $m=2$ for the ANIM and $\rho=0.15, \beta=0.25, p=3.0$ for the FBSN (for more details, see [22]). Especially, the RES will be regarded as 0 if $\mathrm{RES}<10^{-17}$ in our numerical results. 
The initial guess will be selected with $z_{(1)}^{0}=\left[\varepsilon_{0}, q^{\top}\right]^{\top}, z_{(2)}^{0}=(0,0, \cdots, 0)^{\top}$ and $z_{(3)}^{0}=(1,1, \cdots, 1)^{\top}$. Firstly, for Example 4.1, we compare the performance of numerical results of the ANIM with the FBSN and the CBSN by arranging different $n$. From Tables 1-2, we find the ANIM, the FBSN and the CBSN are reliably effective methods. However, from the aspects of iteration number (refer to the overall number of iterations) or CPU, the FBSN and the CBSN do not stand comparison with the ANIM. We also notice that the superiority of convergence performance of the ANIM is distinct as the increase of the problem scale.

Example 4.1 ([27]). We first consider $w:=A u+q$, i.e., the special form of the weakly nonlinear complementarity problem with

$$
A=\operatorname{tridiag}(-1,4,-1), \quad q=(-1, \cdots,-1)^{\top} .
$$

Table 1: Numerical results for Example 4.1.

\begin{tabular}{cccccc}
\hline Initials & & \multicolumn{5}{c}{$z_{(1)}^{0}$} & $z_{(1)}^{0}$ & $z_{(2)}^{0}$ & $z_{(2)}^{0}$ \\
\hline Methods & & ANIM & FBSN & ANIM & FBSN \\
\hline \multirow{2}{*}{$\mathrm{n}=500$} & It & 2 & 11 & 3 & 11 \\
& CPU & 0.1032 & 0.3788 & 0.1511 & 0.4340 \\
& RES & $6.1815 e-016$ & $6.6613 e-016$ & $5.6610 e-016$ & $1.1047 e-015$ \\
& It & 2 & 11 & 3 & 11 \\
$\mathrm{n}=1000$ & CPU & 0.5880 & 1.1551 & 0.8808 & 1.3629 \\
& RES & $6.1815 e-016$ & $6.6613 e-016$ & $5.6610 e-016$ & $1.1047 e-015$ \\
& It & 2 & 11 & 3 & 11 \\
$\mathrm{n}=2000$ & CPU & 3.2900 & 4.2802 & 5.1104 & 5.2537 \\
& RES & $6.1815 e-016$ & $6.6613 e-016$ & $5.6610 e-016$ & $1.1047 e-015$ \\
& It & 2 & 11 & 3 & 11 \\
$\mathrm{n}=2500$ & CPU & 5.9151 & 6.4952 & 6.0723 & 8.0709 \\
& RES & $6.1815 e-016$ & $6.6613 e-016$ & $5.6610 e-016$ & $1.1047 e-015$ \\
\hline
\end{tabular}

Table 2: Numerical results for Example 4.1.

\begin{tabular}{cccccc}
\hline Initials & & $z_{(1)}^{0}$ & $z_{(1)}^{0}$ & $z_{(2)}^{0}$ & $z_{(2)}^{0}$ \\
\hline Methods & & ANIM & CBSN & ANIM & CBSN \\
\hline \multirow{2}{*}{$\mathrm{n}=800$} & It & 2 & 3 & 3 & 4 \\
& CPU & 0.3332 & 0.4838 & 0.4840 & 0.6879 \\
& RES & $6.1815 e-016$ & $2.1678 e-016$ & $5.6610 e-016$ & $2.7616 e-016$ \\
$\mathrm{n}=1500$ & It & 2 & 3 & 3 & 4 \\
& CPU & 1.5395 & 2.3335 & 2.3196 & 3.0597 \\
& RES & $6.1815 e-016$ & $2.1678 e-016$ & $5.6610 e-016$ & $2.7616 e-016$ \\
$\mathrm{n}=3000$ & It & 2 & 3 & 3 & 4 \\
& CPU & 10.4229 & 15.6110 & 15.6472 & 20.6621 \\
& RES & $6.1815 e-016$ & $2.1678 e-016$ & $5.6610 e-016$ & $2.7616 e-016$ \\
$\mathrm{n}=5000$ & It & 2 & 3 & 3 & 4 \\
& CPU & 45.8406 & 65.4892 & 69.7337 & 89.2019 \\
& RES & $6.1815 e-016$ & $2.1678 e-016$ & $5.6610 e-016$ & $2.7616 e-016$ \\
\hline
\end{tabular}

Example 4.2. This problem arises from the discretization of free boundary problems. Let $\Omega=(0,1) \times(0,1)$ and function $g$ satisfy $g(0, y)=y(1-y), g(x, y)=0$ on $y=0, y=1$ or $x=1$. Consider the following problem: find $t$ such that

$$
\begin{cases}t \geqslant 0, & \text { in } \Omega, \\ -\Delta t+f(x, y, t)-8(y-0.5) \geqslant 0, & \text { in } \Omega, \\ t(-\triangle t+f(x, y, t)-8(y-0.5))=0, & \text { in } \Omega, \\ t=g, & \text { in } \partial \Omega\end{cases}
$$


where $f(x, y, t)$ is continuously differentiable and $\frac{\partial f}{\partial t} \geqslant 0$ on $\bar{\Omega} \times\{t: t \geqslant 0\}$.

The numerical results of Example 4.2 are shown by Tables 3-6 and Figures 1-2 for different nonlinear functions and the matrix dimensions. From these tables and figures, we observe that the ANIM is competitive to the FBSN and MMSA for large-scale weakly nonlinear complementarity problems. To sum up, from the results of numerical examples, we demonstrate the efficiency of the ANIM.

Table 3: Numerical results for Example 4.2 with $f(x, y, t)=t^{2}$.

\begin{tabular}{cccccc}
\hline Initials & & $z_{(1)}^{0}$ & $z_{(1)}^{0}$ & $z_{(2)}^{0}$ & $z_{(2)}^{0}$ \\
\hline Methods & & $\mathrm{n}=225$ & $\mathrm{n}=961$ & $\mathrm{n}=2209$ & $\mathrm{n}=3969$ \\
\hline \multirow{2}{*}{ FBSN } & It & 151 & 151 & 151 & 151 \\
& CPU & 4.7936 & 29.2284 & 254.8708 & 1458.9 \\
& RES & $8.2364 e-002$ & $8.3124 e-002$ & $8.342 e-002$ & $8.333 e-002$ \\
& It & 104 & 393 & 853 & 401 \\
MMSA & CPU & 0.0326 & 2.3810 & 26.8192 & 40.3858 \\
& RES & $8.7282 e-013$ & $9.9680 e-013$ & $9.7832 e-013$ & $9.8603 e-013$ \\
& It & 9 & 9 & 2 & 2 \\
ANIM & CPU & 0.0617 & 2.1585 & 3.3131 & 16.6964 \\
& RES & 0 & 0 & 0 & 0 \\
\hline
\end{tabular}

Table 4: Numerical results for Example 4.2 with $f(x, y, t)=t+\sin t$.

\begin{tabular}{cccccc}
\hline Initials & & $z_{(1)}^{0}$ & $z_{(1)}^{0}$ & $z_{(2)}^{0}$ & $z_{(2)}^{0}$ \\
\hline Methods & & $\mathrm{n}=225$ & $\mathrm{n}=961$ & $\mathrm{n}=2209$ & $\mathrm{n}=3969$ \\
\hline \multirow{3}{*}{ FBSN } & It & 151 & 151 & 151 & 151 \\
& CPU & 4.8202 & 31.4063 & 305.5803 & 1539.5 \\
& RES & $9.1410 e-002$ & $9.2369 e-002$ & $9.231 e-002$ & $9.261 e-002$ \\
& It & 105 & 396 & 314 & 404 \\
MMSA & CPU & 0.0317 & 2.3881 & 8.3169 & 36.5056 \\
& RES & $8.0910 e-013$ & $9.4929 e-013$ & $9.2611 e-013$ & $9.8210 e-013$ \\
& It & 7 & 7 & 2 & 2 \\
ANIM & CPU & 0.0498 & 1.5487 & 2.1063 & 11.0249 \\
& RES & $1.1757 e-015$ & $1.0710 e-016$ & 0 & 0 \\
\hline
\end{tabular}

Table 5: Numerical results for Example 4.2 with $f(x, y, t)=\frac{t}{1+t}$.

\begin{tabular}{cccccc}
\hline Initials & & $z_{(3)}^{0}$ & $z_{(3)}^{0}$ & $z_{(2)}^{0}$ & $z_{(2)}^{0}$ \\
\hline Methods & & $\mathrm{n}=225$ & $\mathrm{n}=961$ & $\mathrm{n}=2209$ & $\mathrm{n}=3969$ \\
\hline \multirow{3}{*}{ FBSN } & It & 151 & 151 & 151 & 151 \\
& CPU & 4.8505 & 31.2633 & 263.0305 & 1266.1 \\
& RES & $9.1410 e-002$ & $9.2369 e-002$ & $4.1121 e-002$ & $4.2001 e-002$ \\
& It & 105 & 227 & 299 & 388 \\
MMSA & CPU & 0.0244 & 1.4172 & 9.3599 & 37.8653 \\
& RES & $8.0910 e-013$ & $9.9726 e-013$ & $9.0592 e-013$ & $9.7484 e-013$ \\
& It & 2 & 2 & 2 & 2 \\
ANIM & CPU & 0.0157 & 0.3998 & 2.2060 & 10.6302 \\
& RES & 0 & 0 & 0 & 0 \\
\hline
\end{tabular}


Table 6: Numerical results for Example 4.2 with $f(x, y, t)=\ln (1+t)$.

\begin{tabular}{cccccc}
\hline Initials & & $z_{(3)}^{0}$ & $z_{(3)}^{0}$ & $z_{(2)}^{0}$ & $z_{(2)}^{0}$ \\
\hline Methods & & $\mathrm{n}=225$ & $\mathrm{n}=961$ & $\mathrm{n}=2209$ & $\mathrm{n}=3969$ \\
\hline \multirow{3}{*}{ FBSN } & It & 8 & 9 & 10 & 10 \\
& CPU & 0.0534 & 1.4129 & 13.8041 & 81.8000 \\
& RES & $1.3059 e-008$ & $1.5371 e-007$ & $7.4532 e-008$ & $4.8048 e-007$ \\
& It & 114 & 222 & 297 & 388 \\
MMSA & CPU & 0.0273 & 1.3339 & 9.3883 & 41.4062 \\
& RES & $8.9631 e-013$ & $9.6913 e-013$ & $9.9131 e-013$ & $9.2350 e-013$ \\
& It & 2 & 2 & 2 & 2 \\
ANIM & CPU & 0.0156 & 0.3238 & 2.1165 & 12.1649 \\
& RES & 0 & 0 & 0 & 0 \\
\hline
\end{tabular}

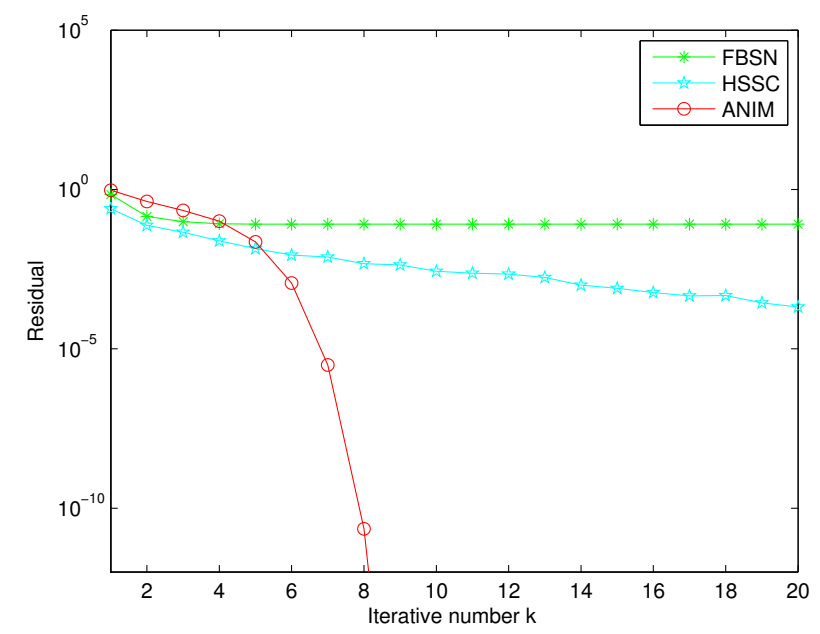

Figure 1: The residuals for Example 4.2 with $n=225, f(x, y, t)=t^{2}$.

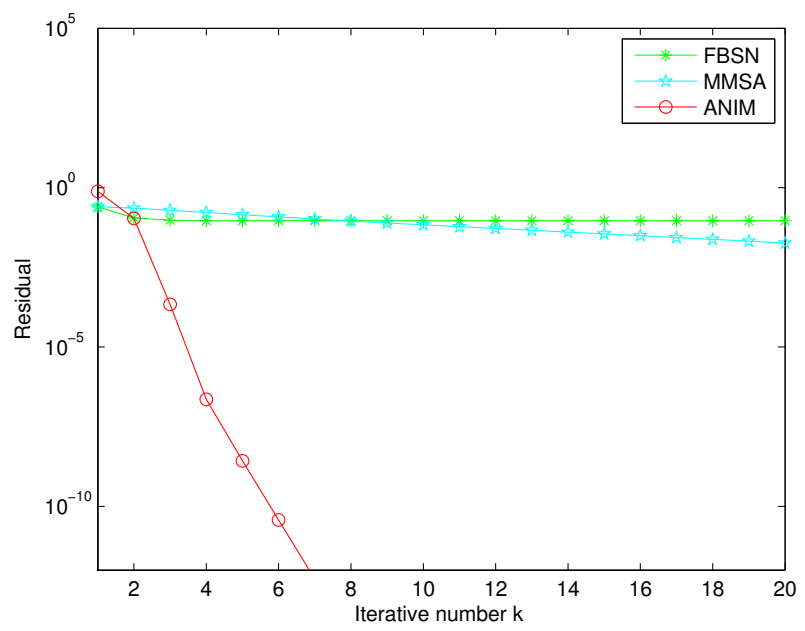

Figure 2: The residuals for Example 4.2 with $n=225, f(x, y, t)=t+\sin t$. 


\section{Conclusion}

In this paper, an accelerated Newton iteration method (ANIM) is proposed for solving a class of largescale weakly nonlinear complementarity problem. Under appropriate assumptions, the ANIM possesses at least $m+1$ order convergence rate. The new technique will remarkably improve the convergence efficiency to solve large-scale weakly nonlinear complementarity problem, which has been shown by the theoretical analysis section in details. As a matter of fact, the strategy can also be deemed as an accelerated process for the classical Newton method. The proposed approach is illustrated by some numerical examples and compared with other popular iteration solvers at present. Numerical test results demonstrate that the method works quite well in practical problem.

\section{Acknowledgment}

The authors thank the anonymous referee for helping to improve the original manuscript by valuable suggestions. The research was supported by Fujian Natural Science Foundation (2015J01578,2016J01005), The Outstanding Talents in The New Century Training Plan of Fujian Province University (15kxtzl3,2015; 17kxtz19,2017), National Postdoctoral Program for Innovative Talents (BX201600182) and China Postdoctoral Science Foundation (2016M600141), Strategic Priority Research Program (B) of the Chinese Academy of Sciences (XDB18010202).

\section{References}

[1] Z.-Z. Bai, On the convergence of the multisplitting methods for the linear complementarity problem, SIAM J. Matrix Anal. Appl., 21 (1999), 67-78. 1

[2] Z.-Z. Bai, Experimental study of the asynchronous multisplitting relaxtation methods for linear complementarity problems, J. Comput. Math., 20 (2002), 561-574. 1

[3] Z.-Z. Bai, Modulus-based matrix splitting iteration methods for linear complementarity problems, Numer. Linear Algebra Appl., 17 (2010), 917-933. 1

[4] Z.-Z. Bai, D. J. Evans, Matrix multisplitting relaxtion methods for linear complementarity problem, Inter. J. Comput. Math., 63 (1997), 309-326. 1

[5] Z.-Z. Bai, D. J. Evans, Matrix multisplitting methods with applications to linear complementarity problems: Parallel synchronous and chaotic methods, Calculateurs Parallelés Réseaux et Systémes Répartis, 13 (2001), 125-151. 1

[6] Z.-Z. Bai, D. J. Evans, Parallel chaotic multisplitting iterative methods for the large spase linear complementarity problem, J. Comput. Math., 19 (2001), 281-292. 1

[7] Z.-Z. Bai, D. J. Evans, Matrix multisplitting methods with applications to linear complementarity problems: Parallel asynchronous methods, Int. J. Comput. Math., 79 (2002), 205-232. 1

[8] Z.-Z. Bai, Y.-G. Huang, A class of asynchronous iterations for the linear complementarity problem, J. Comput. Math., 21 (2003), 773-790. 1

[9] Z.-Z. Bai, L.-L. Zhang, Modulus-based synchronous multisplitting iteration methods for linear complementarity problems, Numer. Linear Algebra Appl., 20 (2013), 425-439. 1

[10] X. Chen, Z. Nashed, L. Qi, Smoothing methods and semismooth methods for nondifferentiable operator equations, SIAM J. Numer. Anal., 38 (2000), 1200-1216. 1

[11] R. W. Cottle, J.-S. Pang, R. E. Stone, The Linear Complementarity Problem, Academic Press, Boston, (1992). 1

[12] C. Cryer, The solution of a quadratic programming using systematic overrelaxation, SIAM J. Control, 9 (1971), 385-392. 1

[13] L. Cvetković, V. Kostić, A note on the convergence of the MSMAOR method for linear complementarity problems, Numer. Linear Algebra Appl., 21 (2014), 534-539. 1

[14] B. C. Eaves, C. E. Lemke, Equivalence of LCP and PLS, Math. Oper. Res., 6 (1981), 475-484. 1

[15] B. C. Eaves, H. Scarf, The solution of systems of piecewise linear equations, Math. Oper. Res., 1 (1976), 1-27. 1

[16] Y. ElFoutayeni, M. Khaladi, Using vector divisions in solving the linear complementarity problem, J. Comput. Appl. Math., 236 (2012), 1919-1925. 1

[17] A. Fischer, A special Newton-type optimization method, Optimization, 24 (1992), 269-284. 4

[18] N. Huang, C.-F. Ma, The modulus-based matrix splitting algorithms for a class of weakly nonlinear complementarity problems, Numer. Linear Algebra Appl., 23 (2016), 558-569. 1, 4

[19] X.-D. Huang, Z.-G. Zeng, Y.-N. Ma, The theory and methods for nonlinear numerical analysis, Wuhan University Press, Wuhan, (2004). 3.1, 3

[20] H.-Y. Jiang, L. Qi, A new nonsmooth equations approach to nonlinear complementarity problems, SIAM J. Control Optim., 35 (1997), 178-193. 1 
[21] C. E. Lemke, Bimatrix equilibrium points and mathematical programming, Management Sci., 11 (1965), 681-689. 1

[22] D. Luca, F. Fancchinei, C. Kanzow, A semismooth equation approach to the solution of nonlinear complementarity problems, Math. Programming, 75 (1996), 407-439. 4

[23] K. G. Murty, Linear Complementarity, Linear and Nonlinear Programming, Heldermann, Berlin, (1988). 1

[24] J.-S. Pang, Newton's method for B-differentiable equations, Math. Oper. Res., 15 (1990), 311-341. 1

[25] L. Qi, J. Sun, A nonsmooth version of Newtons method, Math. Programming, 58 (1993), 353-367. 1

[26] H. Scarf, The approximation of fixed points of a continuous mapping, SIAM J. Appl. Math., 15 (1967), 1328-1343. 1

[27] Z. Sun, J.-P. Zeng, A monotone semismooth Newton type method for a class of complementarity problems, J. Comput. Appl. Math., 235 (2011), 1261-1274. 1, 4.1

[28] P. Tseng, On linear convergence of iterative method for the variational inequality problem, J. Comput. Appl. Math., 60 (1995), 237-252. 1

[29] Z. Yu, Y. Qin, A cosh-based smoothing Newton method for P0 nonlinear complementarity problem, Nonlinear Anal. Real World Appl., 12 (2011), 875-884. 4

[30] L.-L. Zhang, Two-step modulus based matrix splitting iteration for linear complementarity problems, Numer. Algorithms, 57 (2011), 83-99. 1

[31] N. Zheng, J.-F. Yin, Accelerated modulus-based matrix splitting iteration methods for linear complementarity problem, Numer. Algorithms, 64 (2013), 245-262. 1 